\title{
Synchronous Squamous Cell Carcinoma of Tongue and Papillary Carcinoma of Thyroid - A Rare Case Report
}

\author{
Dr. A. N. Aswin ${ }^{1}$, Dr. Arvind Krishnamurthy ${ }^{2}$
}

\begin{abstract}
Synchronous malignancies were traditionally considered difficult to diagnose as one malignancy may be indolent and is usually picked up as an incidental finding while proceeding with staging workup or treatment for the other malignancy. However with advancements in imaging technology, we are able to timely detect and effectively treat such synchronous malignancies. Metastatic papillary carcinoma thyroid in the presence of squamous cell carcinoma of oral cavity is a rare clinical phenomenon. We report an interesting case of one such in a 61-year-old male patient was diagnosed and treated for a locally advanced squamous cell carcinoma of tongue. A synchronous carcinoma of the thyroid gland was subsequently detected and appropriately managed. The patient was finally correctly staged as a synchronous double cancer (Stage 3 carcinoma tongue and "Stage IVA" carcinoma thyroid) which incidentally portended a better prognosis.
\end{abstract}

Keywords: Synchronous, Squamous cell carcinoma, papillary carcinoma thyroid, tongue cancer

\section{Introduction}

Synchronous malignancies are difficult to diagnose as one malignancy may be indolent and are usually picked up as incidental findings while proceeding with staging workup or treatment for the other malignancy. Treating them is a challenge firstly due to a delay in achieving a proper diagnosis and also due to the issues of sequencing the treatment for each of them. We report an interesting case of a patient diagnosed with a synchronous dual malignancy, who while on treatment for one malignancy (carcinoma tongue) was incidentally detected to have another malignancy (carcinoma thyroid) and received appropriate treatment for the same.

\section{Case Report}

A 61-year-old male an ex-smoker and ex-alcoholic with coexisting diabetes mellitus, was referred to our center for further evaluation of an ulcer in the right lateral border of tongue with bilateral cervical adenopathy of 2 months duration. Clinical examination revealed a largeulcer measuring $6 \times 5 \mathrm{~cm}$ in the right lateral border of the tongue and bilateral cervical adenopathy(a significant right Level II $1 \mathrm{~cm}$ node and multiple left level neck nodes. involving II, III, IV, the largest measuring $6 \times 4 \mathrm{~cm}$. A punch biopsy from the tongue was suggestive of a poorly differentiated squamous cell carcinoma. An ultrasound neck confirmed the clinical presence of the bilateral neck nodes and subsequent guided fine needle aspiration cytology from the left cervical lymph node was suggestive of metastatic carcinoma. His chest X-ray was within normal limits. The patient was clinically diagnosed as carcinoma right lateral border of the tongue cT3N3M0(Stage IVB)

After a multidisciplinary board discussion, the patient was offered and received concurrent chemo-radiation (60Gy RT to primary and involved neck nodes using 3 field techniqueswith 3 cycles of chemotherapy with cisplatinum. Response evaluation after 6 weeks revealed a complete response in the primary site and the right level II node. The lymph nodes in the left side of the neck however persisted as was also seen in a CT scan of the neck which showed multiple necrotic left sided cervical nodes. \{Figure 1\}

The patient was subsequently planned for Left modified radical neck dissection (MRND). Intra-operatively the nodes appeared cystic with a black tinge and were hence sent for a frozen section, the report of which suggested the presence of metastatic papillary carcinoma thyroid. A total thyroidectomy and a central compartment neck dissection were added to the left MRND. The post-operative histopathology confirmed the diagnosis of papillary carcinoma of thyroid pT1bN1bM0.

The patient had an uneventful post-operative recovery and was subsequently subjected to 100 millicuries of radioactive Iodine ablation. His post therapy scan showed no evidence of functioning metastases with very minimal residual activity in the thyroid region. $\mathrm{He}$ is presently locally controlled and is on regular follow up for close to a year following his treatment. The patient was finally correctly staged as a synchronous double cancer (Stage 3 carcinoma tongue and Stage IVAcarcinoma thyroid) which incidentally portended a better prognosis. 


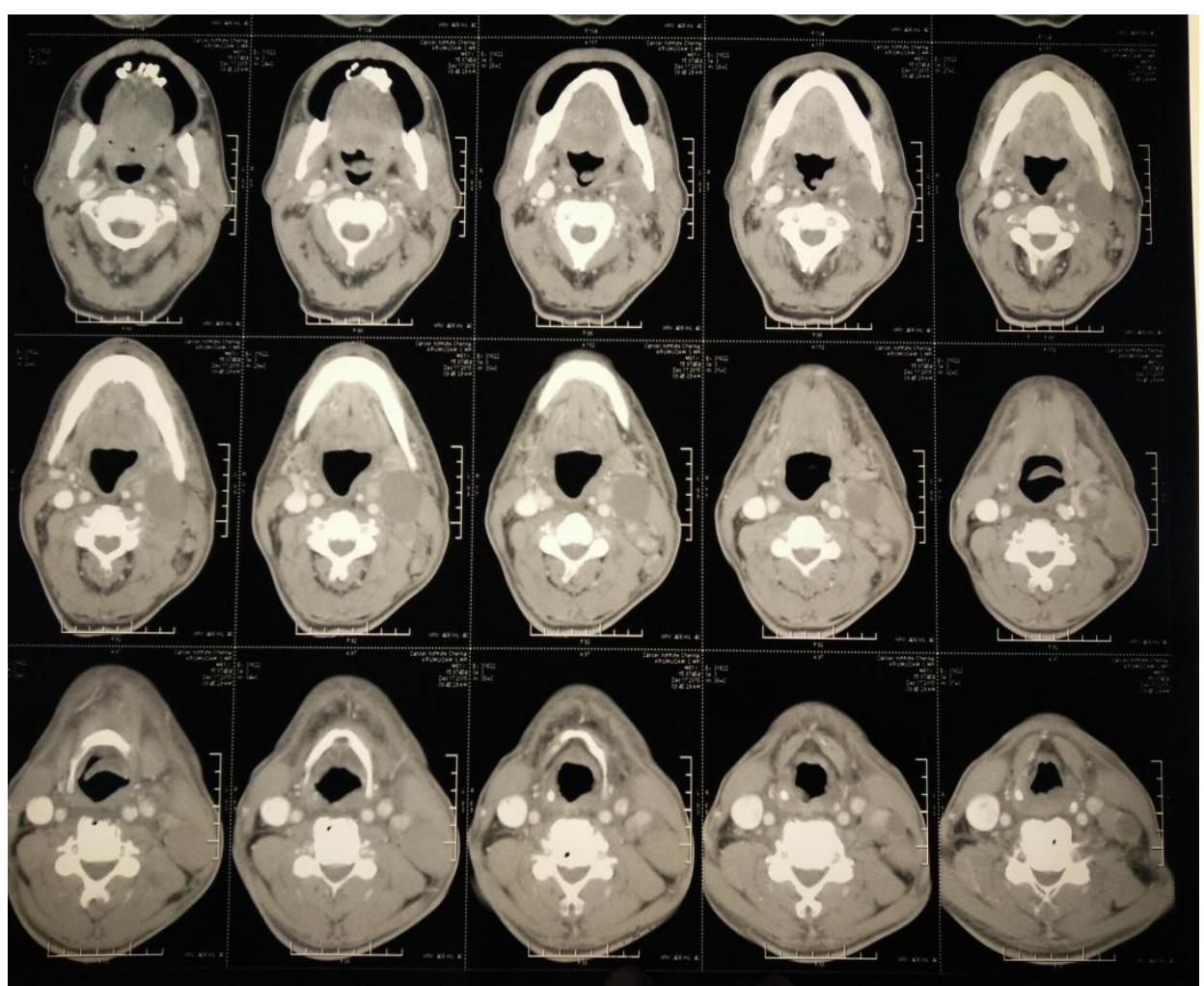

Figure 1: CT Neck showing multiple necrotic left sided cervical adenopathy.

\section{Discussion}

Although synchronous malignancies are rare, it is mandatory to diagnose them at proper time in order to sequence the treatment. Verma et al. reported a similar case with synchronous primaries in the lower alveolus and papillary carcinoma of thyroid. Patient underwent surgery for both the primaries.[1]42 cases of papillary carcinoma thyroid in lymph nodes dissected surgically for treatment of primary squamous cell carcinoma of oral cavity have been reported in the literature. [2] Butler et al. had reported that up to 3\% of patients with head and neck cancer may harbor clinically unsuspected thyroid cancer. [3]Vassiloplou-Sellin and Weber found the incidence of incidental thyroid metastasis to cervical nodes was $0.3 \%[4]$ Warren and Gates have proposed some criteria for the definition of the second primary cancer: tumors have to be malignant and this have to be histologically confirmed, tumors must be separated from normal tissue, non-neoplastic and it must be eliminated the possibility that the second primary tumor to be the metastasis of the index tumor. [5-7] In 1953, Field cancerization was first described as histologically altered epithelium surrounding tumor samples from upper aerodigestive tract.[8] Although field cancerization is very common in head and neck region, two synchronous primary cancers in anatomically distinct organs involving thyroid and tongue is a rare phenomenon.

In our patient, the fine needle aspiration cytology from the cervical node was suggestive of metastatic carcinoma but did not suggest a possible thyroid primary. The patient was hence upstaged to a very advanced tongue primary (Stage IV B), otherwise the stage would have been cT2N1M0 (Stage III). A PET-CTscan for staging would have perhaps picked up the nodules in the thyroid, which was incidentally missed in the ultrasound of the neck. A PET CT scan is considered an option in the staging evaluation of any patient with locally advanced head and neck cancers. It may be considered preferable in cases where in primary in one side and more significant nodes on the contralateral side, even if other investigations are normal.

\section{Conclusion}

With advancements in technology, we are able to detect synchronous malignancies and able to treat them effectively.In the presence of two primaries, the one which is more aggressive should be treated first followed by the less aggressive one. Metastatic papillary carcinoma thyroid in the presence of squamous cell carcinoma of oral cavity is a rare clinical phenomenon and clinicians should be aware of such a rare differential.

\section{References}

[1] Verma R, Tripathi N, Aggarwal P, Panda N. Metastatic papillary carcinoma thyroid co-existing with oral cavity squamous cell carcinoma: A case report and review of literature. Egyptian Journal of Ear, Nose, Throat and Allied Sciences. 2014;15(2):125-128.

[2] Altumbabic H, Salkic A.Incidental metastasis of papillary thyroid carcinoma in lymph nodes of patient with squamous cell carcinoma of oral cavity: a Case Report.Acta Med Sal.2010; 39(2):104-106.

[3] Butler J, Tulinius H, Ibanez M, Ballantyne A, Clark R. Significance of thyroid tissue in lymph nodes associated with carcinoma of the head, neck or lung. Cancer. 1967;20(1):103-112.

[4] Vassilopoulou-Sellin R, Weber R. Metastatic thyroid cancer as an incidental finding during neck dissection: 


\section{International Journal of Science and Research (IJSR) \\ ISSN (Online): 2319-7064}

Index Copernicus Value (2015): 78.96 | Impact Factor (2015): 6.391

Significance and management. Head Neck. 1992;14(6):459-463.

[5] Choy A, Andrew Hasselt C, Chisholm E, Williams S, King W, Li A. Multiple primary cancers in Hong Kong Chinese patients with squamous cell cancer of the head or neck. Cancer. 1992;70(4):815-820.

[6] Jones A, Morar P, Phillips D, Field J, Husband D, Helliwell T. Second primary tumors in patients with head and neck squamous cell carcinoma.Cancer. 1995;75(6):1343-1353.

[7] Braakhuis B. Second Field Tumors: A New Opportunity for Cancer Prevention?.The Oncologist. 2005;10(7):493-500.

[8] Slaughter D, Southwick H, Smejkal W. "Field cancerization" in oral stratified squamous epithelium. Clinical implications of multicentric origin.Cancer. 1953;6(5):963-968.

[9] Quon A, Fischbein NJ, McDougall IR, Le QT, Loo BW Jr, Pinto H, et al. Clinical role of 18F-FDG PET/CT in the Management of squamous cell carcinoma of the head and neck and thyroid carcinoma. J Nucl Med. 2007;48(Suppl 1):58S-67S

[10] Tokuchi Y, Kamachi M, Harada M, Hasegawa M, Mlshina $\mathrm{T}$, Yamashiro $\mathrm{K}$ et al. Synchronous Triple Lung Cancers after Treatment for Non-Hodgkin's Lymphoma: Metachronous Quadruple Cancers. Intern Med. 2003;42(10):1031-1034. 